\title{
Duplication of chromosome segment 12q13-15 in a lipomatous tumor with minimal nuclear atypia: A case report
}

\author{
JUN NISHIO ${ }^{1}$, HIROSHI IWASAKI ${ }^{2}$, TERUFUMI SHIBATA ${ }^{1}$, KAZUKI NABESHIMA ${ }^{2}$ and MASATOSHI NAITO ${ }^{1}$ \\ Departments of ${ }^{1}$ Orthopaedic Surgery and ${ }^{2}$ Pathology, Faculty of Medicine, Fukuoka University, Fukuoka 814-0180, Japan
}

Received May 31, 2014; Accepted March 6, 2015

DOI: $10.3892 / 01.2016 .4305$

\begin{abstract}
Ordinary lipoma is cytogenetically characterized by structural rearrangements, particularly translocations, of 12q13-15. By contrast, atypical lipomatous tumors exhibit supernumerary ring or giant marker chromosomes that are composed mainly of amplified material from the 12q13-15 chromosome segment. The present study describes the cytogenetic and molecular cytogenetic findings from a lipomatous tumor with minimal nuclear atypia that was identified in a 49-year-old female patient. Magnetic resonance imaging of the right shoulder revealed a $13-\mathrm{cm}$ fatty mass in the subcutaneous layer that possessed only pencil-line septa. Contrast-enhanced fat-suppressed T1-weighted images demonstrated faint enhancement. A marginal excision was performed. Histologically, the tumor was composed of lobules that consisted of mature adipocytes separated by thin fibrous septa. There was minimal nuclear atypia in certain cells, and a small number of binucleated cells were also observed within the tumor. Immunohistochemically, the tumor cells did not reveal the expression of murine double-minute 2 (MDM2). Cytogenetic analysis revealed a complex karyotype with several numerical and structural alterations, including $12 \mathrm{q}$ rearrangements. Spectral karyotyping demonstrated a duplication of chromosome segment 12q13-15. Interphase fluorescence in situ hybridization analysis revealed no MDM2 gene amplification. The present case indicates that duplication of $12 \mathrm{q}$ may be associated with minimal nuclear atypia in a subset of lipomatous tumors.
\end{abstract}

\section{Introduction}

Lipomatous tumors constitute the largest subgroup of mesenchymal neoplasms and are commonly encountered in clinical practice. The diagnosis of lipomatous tumors is primarily based on clinical features and histological patterns (1).

Correspondence to: Dr Jun Nishio, Department of Orthopaedic Surgery, Faculty of Medicine, Fukuoka University, 7-45-1 Nanakuma, Jonan-ku, Fukuoka 814-0180, Japan

E-mail: jnishio@cis.fukuoka-u.ac.jp

Key words: lipomatous tumor, chromosome 12q13-15, duplication, spectral karyotyping, murine double-minute 2
Previously, immunostaining for murine double-minute 2 (MDM2), cyclin-dependent kinase 4 (CDK4), and p16 has been demonstrated to be a highly sensitive and specific method for distinguishing between atypical lipomatous tumors (ALTs) and other lipomatous tumors (2).

Cytogenetic and molecular genetic studies have provided insight into the pathogenesis of a variety of lipomatous tumors. Overall, $\sim 75 \%$ of ordinary lipomas harbor chromosomal rearrangements of $12 q 13-15$, resulting in deregulation of the high mobility group AT-hook 2 (HMGA2) gene (3). By contrast, the cytogenetic hallmark of ALT is the presence of one or more supernumerary ring or giant marker chromosomes (4). The rings and giant markers invariably contain amplified sequences derived from chromosome 12q13-15 (5), including the MDM2 and CDK4 genes.

Several cases of lipomatous tumors with minimal nuclear atypia and low-level gain of the $12 q 13-15$ region have been reported (6-8). The present study describes the cytogenetic and molecular cytogenetic findings of a lipomatous tumor with minimal nuclear atypia arising in the shoulder of a 49-year-old woman. Written informed consent for this study was obtained from the patient.

\section{Case report}

A 49-year-old woman presented to Fukuoka University Hospital (Fukuoka, Japan) with a slow-growing, painless mass in the right shoulder that had been developing over the previous ten years. Physical examination revealed a 13-cm, soft, mobile, non-tender mass. The neurovascular examinations were unremarkable. The laboratory values were within normal ranges. Magnetic resonance imaging (MRI) revealed a well-defined subcutaneous mass. The mass exhibited a signal intensity identical to that of subcutaneous fat on T1-weighted sequences (Fig. 1A) and was completely suppressed on T2-weighted short tau inversion recovery (STIR) sequences (Fig. 1B). Faint hyperintensity within the mass was also observed on T2-weighted STIR sequences. Contrast-enhanced fat-suppressed T1-weighted sequences demonstrated faint enhancement (Fig. 1C). A marginal excision was therefore performed.

Microscopically, the tumor was composed of lobules of mature adipocytes separated by delicate thin fibrous septa (Fig. 2A). There was minimal nuclear atypia in certain cells (Fig. 2B), and a small number of binucleated cells were 
also observed. Immunohistochemically, the tumor cells were revealed to not express MDM2. The pathological diagnosis was lipomatous tumor with minimal nuclear atypia.

A representative fresh tissue sample was obtained for cytogenetic analysis. Standard culture and harvest procedures were performed, as previously described (9). The karyotypes were expressed according to the International System for Human Cytogenetic Nomenclature 2009 (10). In total, 20 metaphase cells were routinely analyzed. Conventional cytogenetic analysis revealed a complex karyotype with several numerical and structural alterations, including 12q rearrangements (Fig. 3). The karyotype was identified as 46,XX,add(1)(q42),-3, ins(12;?)(q15;?),ins(13;?)(q14;?),-15,+mar1,+mar2(20).

Spectral karyotyping (SKY) analysis was performed on unstained cytogenetic preparations, according to the manufacturer's instructions (Applied Spectral Imaging, Carlsbad, CA, USA) and previous descriptions (11). Spectral images were acquired using an SD200 spectral bio-imaging system (Applied Spectral Imaging) and analyzed using the SkyView software (Applied Spectral Imaging). In total, five metaphase cells were analyzed. SKY analysis revealed that the aberrations were more complex than was demonstrated by G-banding analysis. Based on the combined results, the karyotype was reinterpreted as follows: 46,XX,der(1)t(1;3)(q42;p21), $\operatorname{der}(3) \mathrm{t}(1 ; 3) \operatorname{del}(3)(\mathrm{q} 13), \operatorname{dup}(12)(\mathrm{q} 15 \mathrm{q} 13), \operatorname{der}(13) \mathrm{t}(13 ; 15)(\mathrm{q} 12 ; \mathrm{q} 24)$, $\operatorname{der}(15) \mathrm{t}(3 ; 15)(? ; \mathrm{q} 21) \mathrm{t}(3 ; 13)(? ; \mathrm{q} 12)$ (Fig. 4).

Fluorescence in situ hybridization (FISH) was performed on paraffin-embedded tissue sections using a Poseidon Repeat-Free MDM2 (12q15)/SE12 control probe (Kreatech Diagnostics, Amsterdam, Netherlands). Briefly, the 4- $\mu \mathrm{m}$ thick paraffin-embedded tissue sections were deparaffinized, dehydrated and incubated with pepsin, according to the manufacturer's instructions. The probe and slides were co-denatured at $80^{\circ} \mathrm{C}$ for $5 \mathrm{~min}$ and incubated overnight at $37^{\circ} \mathrm{C}$ in a humidified chamber. Post-hybridization washing was performed following standard procedures. The slides were counterstained with DAPI. Overall, $\geq 200$ interphase nuclei were scrutinized. An MDM2/SE12 ratio >2.0 was considered amplified, whereas a ratio $\leq 2.0$ was considered non-amplified. The interphase FISH analysis revealed no MDM2 gene amplification (data not shown).

The post-operative course was uneventful, and the patient continues to experience good health without evidence of local recurrence at four months post-surgery.

\section{Discussion}

Cytogenetic analysis of lipomatous tumors has revealed that the various histological subtypes are characterized by distinctive clonal chromosomal abnormalities (1). Ordinary lipomas harbor translocations of 12q13-15, loss of 13q or rearrangements of $6 \mathrm{p} 21$ (3). The most frequent translocation is $\mathrm{t}(3 ; 12)$ (q27-28; $1313-15)$, observed in $25 \%$ of cases with 12q13-15 alterations (12). HMGA2 (12q14) is the target gene of copy number alterations and rearrangements/gene fusions in ordinary lipomas with 12q13-15 aberrations (3). By contrast, ALTs possess supernumerary ring or giant marker chromosomes, typically as the sole anomaly or concomitant with a small number of other numerical or structural aberrations (4). These abnormal chromosomes are mainly composed
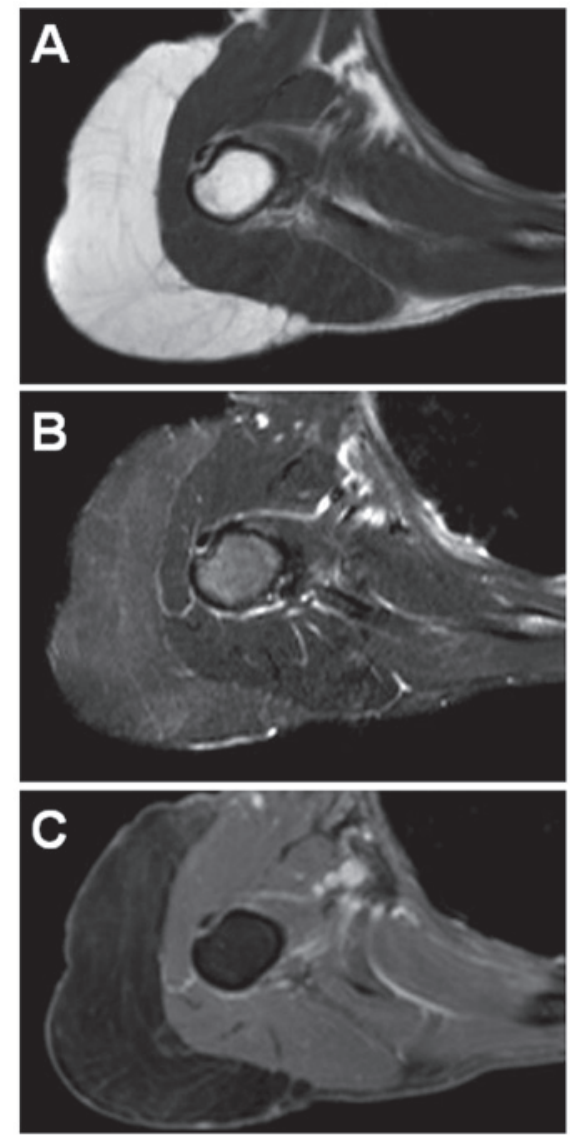

Figure 1. Axial magnetic resonance images of a lipomatous tumor involving the right shoulder. (A) T1-weighted sequence exhibiting a well-defined mass with signal intensity identical to that of subcutaneous fat. (B) The mass is completely suppressed on T2-weighted short tau inversion recovery sequence. Faint hyperintensity within the mass is also observed. (C) Contrast-enhanced fat-suppressed T1-weighted sequence demonstrating faint enhancement.

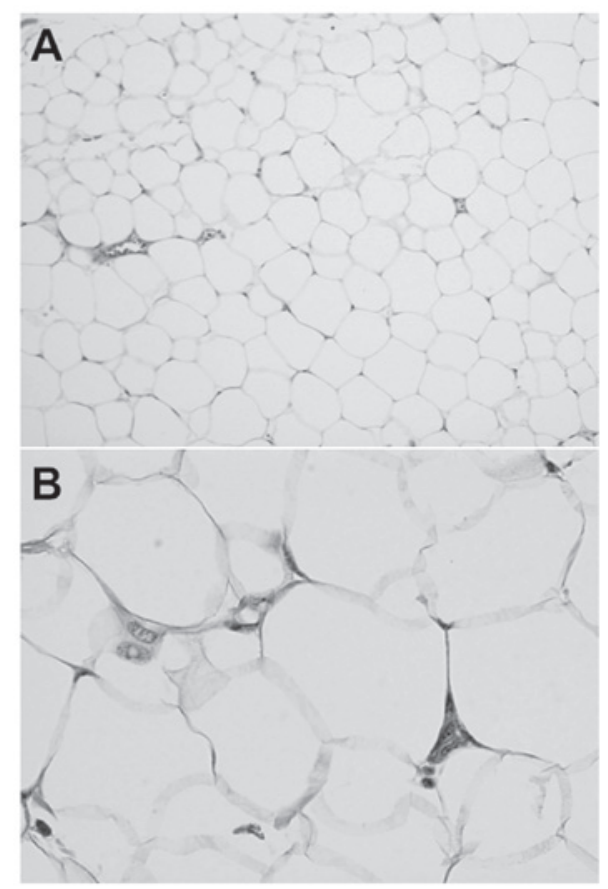

Figure 2. Histological findings of a lipomatous tumor. (A) The tumor consists of mature fat cells exhibiting only a slight variation in cellular size and shape. (B) Minimal nuclear atypia, including slight nuclear enlargement, were identified. 


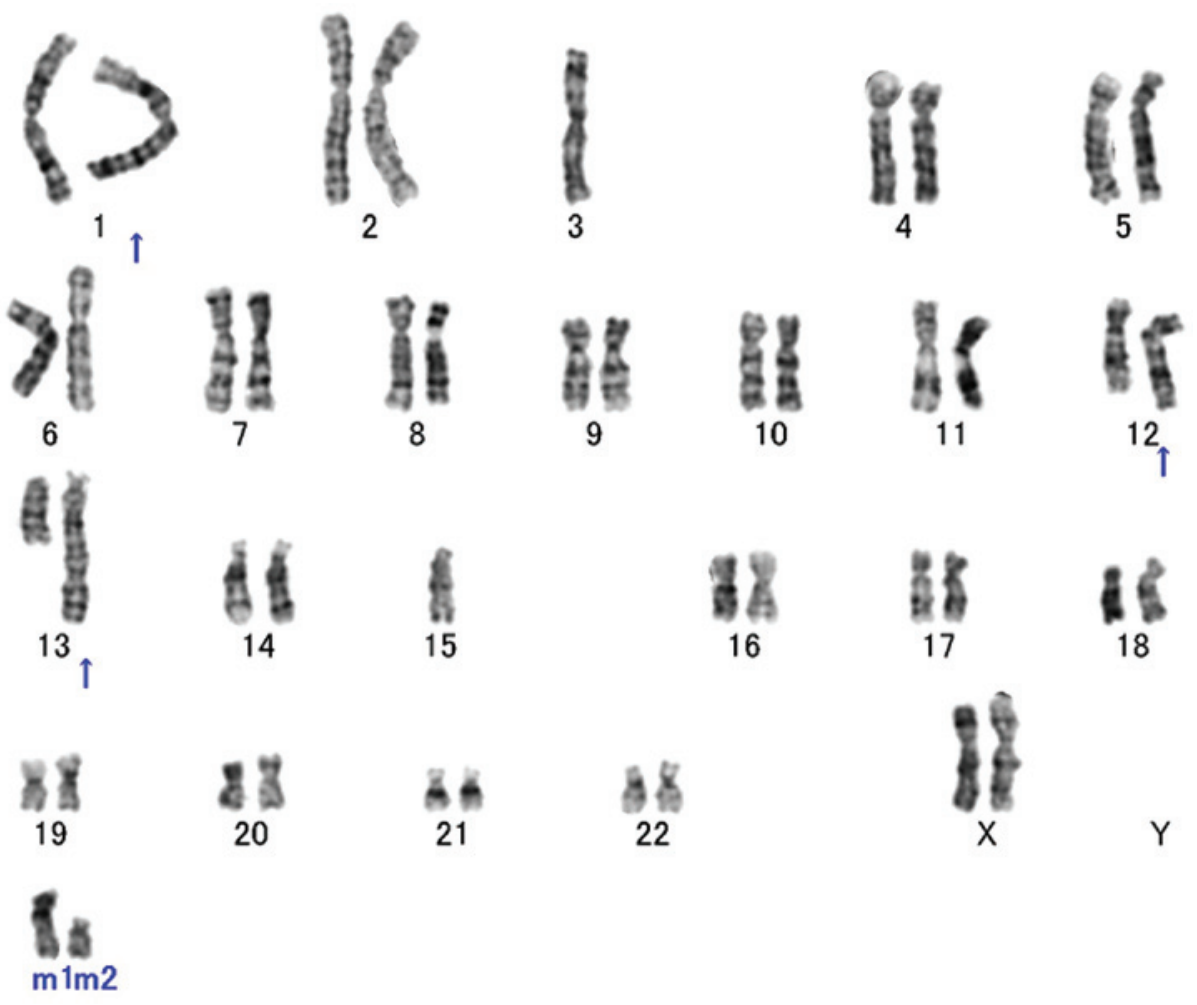

Figure 3. A representative G-banded karyotype of a lipomatous tumor with minimal nuclear atypia. The karyotype is 46,XX,add(1)(q42),-3,ins(12;?)(q15;?), ins(13;?)(q14;?),-15,+mar1,+mar2. Arrows indicate the structural chromosomal aberrations.

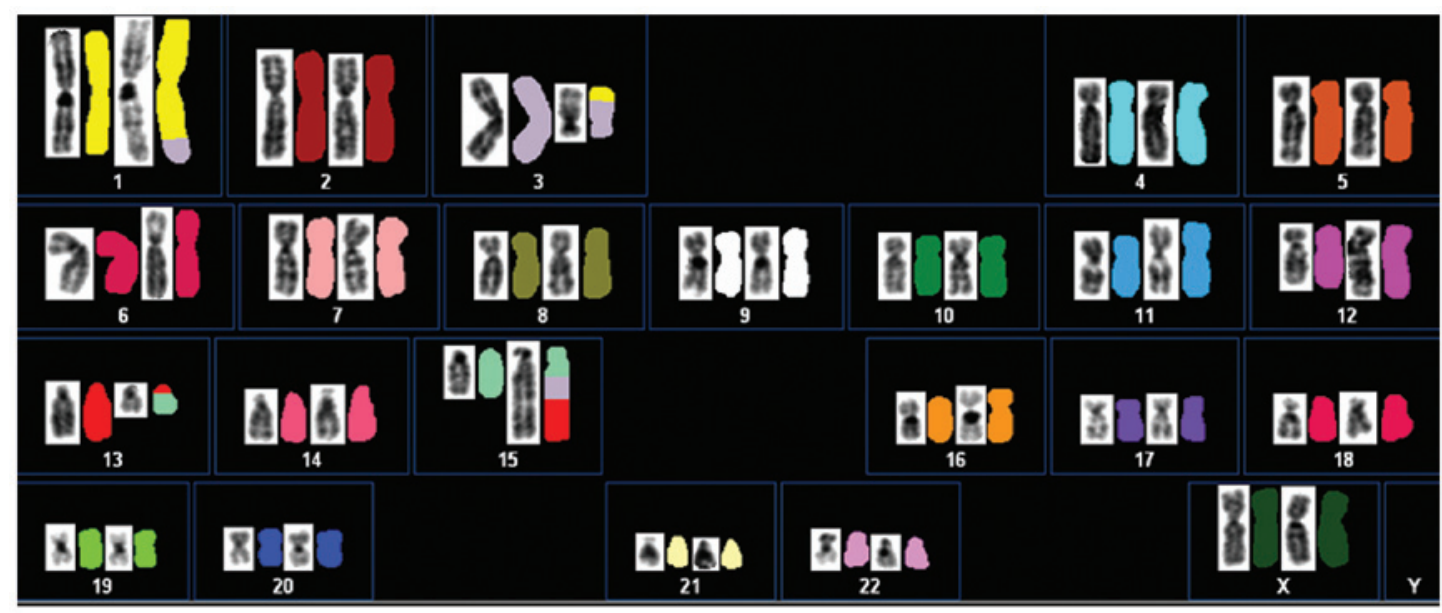

Figure4.Spectralkaryotyping(SKY)ofalipomatoustumorwithminimalnuclearatypia.TheSKYkaryotypeis 46,XX,der(1)t(1;3)(q42;p21),der(3)t(1;3)del(3)(q13), $\operatorname{dup}(12)(\mathrm{q} 15 \mathrm{q} 13), \operatorname{der}(13) \mathrm{t}(13 ; 15)(\mathrm{q} 12 ; \mathrm{q} 24), \operatorname{der}(15) \mathrm{t}(3 ; 15)(? ; \mathrm{q} 21) \mathrm{t}(3 ; 13)(? ; \mathrm{q} 12)$. The classified image is presented alongside the reverse DAPI-banded image.

of amplified sequences of 12q13-15 (5). MDM2 (12q15) is consistently amplified and overexpressed in ALTs, but not in lipomas and is thought to be the main driver gene of the $12 \mathrm{q}$ amplicon (4). The present case demonstrated only a minor degree of nuclear atypia and failed to reveal amplification and overexpression of the $M D M 2$ gene.

In the present study, a duplication of chromosome segment 12q13-15 was identified by SKY. There are extremely few studies reporting lipomatous tumor with this chromosomal aberration $(6,8)$. Mandahl et al (6) suggested that duplication of $12 q$ may be sufficient for the development of minimal nuclear atypia and formation of ALTs. Italiano et al (8) revealed the existence of a genetic and morphological continuum between ordinary lipomas and ALTs. Storlazzi et al (7) described the case of a patient with atypical lipomatous tumor that demonstrated minimal nuclear atypia and was cytogenetically characterized by the consistent presence of 1-3 supernumerary rings and low-level amplification of the MDM2 gene. Overall, these observations indicate the existence of a subset of lipomatous tumors that are characterized by minimal nuclear atypia and gain or low-level amplification of 12q sequences.

Deletions or structural rearrangements of the long arm of chromosome 13 have been observed in benign and intermediate lipomatous tumors (13). In the present study, complex 
rearrangements of $13 \mathrm{q} 12$ were identified. The HMG family gene high mobility group box $1(H M G B I)$ has been identified as located at this chromosomal region. Kazmierczak et al (14) revealed that there are no intragenic rearrangements of $H M G B 1$ in lipomas with 13q12 aberrations. By contrast, Petit et al (15) revealed a novel HMGA2-lipoma HMGIC fusion partner fusion gene in a lipoma that possessed a karyotype of $\mathrm{t}(12 ; 13)$ (q13-15;12). Additional studies are required to elucidate the significance of this cytogenetic alteration in the development of lipomatous tumors.

In summary, the present study described a unique case of lipomatous tumor with minimal nuclear atypia and duplication of 12q13-15. The present and previously reported studies suggest that the degree of nuclear atypia may be associated with the level of chromosome $12 \mathrm{q}$ amplification in lipomatous tumors.

\section{Acknowledgements}

This study was supported in part by the Foundation for the Promotion of Medical Science and JSPS KAKENHI (grant no. 25462355).

\section{References}

1. Nishio J: Contributions of cytogenetics and molecular cytogenetics to the diagnosis of adipocytic tumors. J Biomed Biotechnol 2011: 524067, 2011.

2. Thway K, Flora R, Shah C, Olmos D and Fisher C: Diagnostic utility of p16, CDK4, and MDM2 as an immunohistochemical panel in distinguishing well-differentiated and dedifferentiated liposarcomas from other adipocytic tumors. Am J Surg Pathol 36: 462-469, 2012

3. Bartuma H, Hallor KH, Panagopoulos I, Collin A, Rydholm A, Gustafson P, Bauer HC, Brosjö O, Domanski HA, Mandahl N and Mertens F: Assessment of the clinical and molecular impact of different cytogenetic subgroups in a series of 272 lipomas with abnormal karyotype. Genes Chromosomes Cancer 46 : 594-606, 2007.
4. Dei Tos AP and Pedeutour F: Atypical lipomatous tumour. In: World Health Organization Classification of Tumours of Soft Tissue and Bone. Fletcher CDM, Bridge JA, Hogendoorn PCW and Mertens F (eds). IARC Press, Lyon, pp33-36, 2013.

5. Pedeutour F, Forus A, Coindre JM, et al: Structure of the supernumerary ring and giant rod chromosomes in adipose tissue tumors. Genes Chromosomes Cancer 24: 30-41, 1999.

6. Mandahl N, Akerman M, Aman P, et al: Duplication of chromosome segment $12 \mathrm{q} 15-24$ is associated with atypical lipomatous tumors: A report of the CHAMP collaborative study group. CHromosomes And MorPhology. Int J Cancer 67: 632-635, 1996.

7. Storlazzi CT, Mertens F, Domanski H, Fletcher CDM, Wiegant J and Mandahl N: Ring chromosomes and low-grade gene amplification in an atypical lipomatous tumor with minimal nuclear atypia. Int J Oncol 23: 67-71, 2003.

8. Italiano A, Cardot N, Dupré F, Monticelli I, Keslair F, Piche M, Mainguené C, Coindre JM and Pedeutour F: Gains and complex rearrangements of the 12q13-15 chromosomal region in ordinary lipomas: The "missing link" between lipomas and liposarcomas? Int J Cancer 121: 308-315, 2007.

9. Nishio J, Aoki M, Nabeshima K, Iwasaki $\mathrm{H}$ and Naito M: Cytogenetic and molecular cytogenetic findings in giant dedifferentiated liposarcoma of the thigh. Oncol Rep 27: 764-768, 2012

10. Shaffer LG, Slovak ML and Campbell LJ (eds): ISCN (2009): An International System for Human Cytogenetic Nomenclature. S Karger, Basel, 2009.

11. Nishio J, Aoki M, Nabeshima $\mathrm{K}$, Iwasaki $\mathrm{H}$ and Naito $\mathrm{M}$ : Characterization of giant marker and ring chromosomes in a pleomorphic leiomyosarcoma of soft tissue by spectral karyotyping. Oncol Rep 28: 533-538, 2012.

12. Sandberg AA: Updates on the cytogenetics and molecular genetics of bone and soft tissue tumors: Lipoma. Cancer Genet Cytogenet 150: 93-115, 2004.

13. Dahlén A, Debiec-Rychter M, Pedeutour F, Domanski HA, Höglund M, Bauer HC, Rydholm A, Sciot R, Mandahl N and Mertens F: Clustering of deletions on chromosome 13 in benign and low-malignant lipomatous tumors. Int J Cancer 103: 616-623, 2003.

14. Kazmierczak B, Dal Cin P, Meyer-Bolte K, Van den Berghe H and Bullerdiek J: HMG1 is not rearranged by $13 \mathrm{q} 12$ aberrations in lipomas. Genes Chromosomes Cancer 24: 290-292, 1999.

15. Petit MM, Schoenmakers EF, Huysmans C, Geurts JM, Mandahl N and Van de Ven WJ: LHFP, a novel translocation partner gene of HMGIC in a lipoma, is a member of a new family of LHFP-like genes. Genomics 57: 438-441, 1999. 